\title{
Impacto da Excisão do Pterígio na refração objetiva: Qual o melhor momento para a correção refrativa?
}

\author{
Pterygium excision impact in objective refraction: when is the best time to glasses pre- \\ scription? \\ El impacto de la Excisión del Pterigio en la refracción objetiva: ¿Cuál es el mejor momento \\ para la corrección refractiva?
}

Paula Renata Caluff Tozzatti ${ }^{1}$, Nathalia Pereira Scherer ${ }^{2}$, Paula Caroline Coelho Fonseca ${ }^{1 \star}$, Rafael Scherer ${ }^{1}$, Fernanda Braga Cordeiro Franco Rodrigues ${ }^{1}$, Graziella de Assis Malerba ${ }^{1}$, Olga Ten Caten Pies Lameira ${ }^{1}$.

\begin{abstract}
RESUMO
Objetivo: Identificar diferenças refratométricas antes e após a exérese de pterígio e o período aproximado para estabilização do grau e acuidade visual em pacientes do Hospital Universitário Bettina Ferro de Souza (HUBFS) no período de abril a agosto de 2017, correspondendo ao melhor momento para a correção óptica. Método: Foi avaliado a acuidade visual, grau e localização do pterígio de 38 pacientes submetidos a exérese de pterígio no HUBFS, antes e após 1, 7 e 30 dias pós cirurgia. A coleta de dados foi realizada por meio de aplicação de questionário sócio-demográfico e formulário para preenchimento médico no pré-operatório e pós-operatório mediante ao preenchimento prévio do termo de consentimento livre e esclarecido (TCLE). Resultados: Observou-se que os pacientes tiveram o seguinte perfil sócio-epidemiológico: idade média de $45,8 \pm 13,9$ anos, sendo $52,6 \%$ mulheres, de localização nasal $(84,2 \%)$, com predomínio daqueles com Graus II e III, por fim somente 7,9\% eram pterígios recidivados, além disso, os pterígios de graus III e IV tiveram maior variação em seu perfil refratométrico após a excisão, chegando a uma variação média máxima de 6,88 dioptrias de equivalente esférico. Conclusão: $O$ período menor que 30 dias após a exérese do pterígio não é o suficiente para receitar óculos devido a grande variação na refratometria nos pacientes pós cirúrgicos.
\end{abstract}

Palavras-chave: Pterígio, Refração Ocular, Amazônia.

\begin{abstract}
Objective: The authors aim to identify refractometry differences before and after pterygium excision and the approximate period for stabilization of the eye refraction and visual acuity in patients from the Hospital Universitário Bettina Ferro de Souza from April to August 2017, corresponding to the best time for the refractive correction. Methods: The visual acuity, degree and location of the pterygium of 38 patients submitted to pterygium excision in the HUBFS were evaluated before and after 1, 7- and 30-days post-surgery. Data collection was performed through the application of a socio-demographic questionnaire and a form for medical completion in the preoperative and postoperative periods by means of the previous completion of the informed consent form. Results: It was observed that the patients had the following socio-epidemiological profile: mean age of $45.8 \pm 13.9$ years, $52.6 \%$ were women, with a nasal location $(84.2 \%)$, with a predominance of those with Degrees II and III, only $7.9 \%$ were recurrent pterygium; in addition, the pterígios of degrees III and IV had greater variation in their refratometric profile after excision, reaching a maximum average variation of 6.88 diopters of spherical equivalent. Summary: The period less than 30 days after the excision of the pterygium is not enough to prescribe glasses due to a large variation in refractometry in post-surgical patients.
\end{abstract}

Keywords: Pterygium, Ocular Refraction, Amazon.

\footnotetext{
${ }^{1}$ Universidade Federal do Pará - UFPA, PA, Brasil. *E-mail: paulacoelho.f@hotmail.com

2Universidade do Estado do Pará- UEPA, PA, Brasil.
}

SUBMETIDO EM: 12/2018

ACEITO EM: 1/2019

PUBLICADO EM: 5/2019

REAS/EJCH | Vol.Sup.22 | e847 | DOI: https://doi.org/10.25248/reas.e847.2019 Página 1 de 9 


\section{RESUMEN}

Objetivo: Identificar las diferentes características refratométricas antes y después del desarrollo y la duración de la actividad visual y de los pacientes en el Hospital Universitário Bettina Ferro de Souza (HUBFS) en abril de 2017 a agosto de 2017, correspondiente a la fecha de la cita óptica. Métodos: Ha sido evaluado la acuidad visual, ubicación y localización del pacto de 38 pacientes submetidos a exérese de pterigión en el HUBFS, antes y após 1,7 e 30 dias pós cirurgía. La coleta de los dados ha sido por medio de la aplicación del cuestionario sócio-demográfico y formulário para el prédito médico no pré-operatório e pós- operatório a través de preenchimento prévio del termo de consentimiento libre y ilustrado. Resultados: Ha sido encontrado que los pacientes se encuentran en el perfil epidemiológico: la media de $45,8 \pm 13,9$ años, con $52,6 \%$ mulheres, de localización nasal $(84,2 \%)$, con predominio de los con Graus II y III, por fín somente, $7,9 \%$ eran pterigión recidivados, además, los pterigións de graus III y IV tuvieron mayor variación en su perfil refractométrico en la parte superior, llegando a una variable media máxima de 6,88 dioptrias de máximo contenido. Conclusión: Un período menor que 30 días después de haber realizado la cirurgia de pterigión no es suficiente para recibir óculos por su gran variación en la refracción y de los pacientes pós cirúrgicos.

Palavras-chave: Pterigión, Refracción Ocular, Amazonia.

\section{INTRODUÇÃO}

O pterígio é uma lesão fibrovascular, causada por uma degeneração fibroelástica da conjuntiva, com aderência corneana, causando prejuízo visual devido alterações biomecânicas da córnea, além de alterações no filme lacrimal. A excisão cirúrgica da lesão pode levar à reconstrução de uma superfície normal, resultando em uma melhora da acuidade visual, principalmente em pterígios mais avançados, nos quais ocorre redução do astigmatismo previamente encontrado (KUJUR $R$ et al., 1999).

Um estudo realizado na Amazônia revelou uma prevalência de pterígio de 15,8\%, principalmente em pessoas com exposição solar intensa nos primeiros 10 anos de vida (COUTTS SJ, COOMBES A, 2000). Além da exposição crônica aos raios ultravioleta, a baixa umidade, a poeira e fatores genéticos têm sido apontados como fatores de risco para o desenvolvimento da patologia (KUJUR R et al., 1999; MISRA S et al., 2014).

Além de erro refracional, o pterígio causa uma redução da sensibilidade ao contraste, mesmo quando a acuidade visual na tabela de Snellen é pouco afetada (KUJUR R et al., 1999). O pterígio pode causar grande desconforto, como sensação de olho seco, corpo estranho, ardência e dor ocular. A única conduta disponível até o momento para a sua resolução é a remoção cirúrgica. Entretanto, a cirurgia está indicada nos casos em que há prejuízo da acuidade visual, limitação da motilidade ocular, inflamações crônicas, sintomas persistentes e alterações grosseiras da estética ocular (KUJUR R et al., 1999; CHOURASIA P et al., 2014).

Estudos apontam a ocorrência de alterações refrativas induzidas pelo pterígio, correlacionando a extensão da lesão sobre a córnea e o valor do astigmatismo corneano, além do seu impacto em outros parâmetros oftalmológicos, como a correção parcial das alterações ceratométricas após a sua exérese (GUMUS K et al., 2011). É preciso observar, também, que a própria cirurgia para a remoção do pterígio pode causar alterações refrativas, pela alteração na regularidade e na simetria corneana (FERRAZ FHS et al., 2002).

Deste modo, o trabalho tem como objetivo principal identificar diferenças refratométricas objetivas antes e após a exérese de pterígio, bem como o período aproximado para estabilização do grau e acuidade visual em pacientes do Hospital Universitário Bettina Ferro de Souza no período de abril a agosto de 2017, correspondendo ao melhor momento para a correção óptica.

\section{MÉTODO}

A pesquisa, de perspectiva local, foi realizada no Hospital Universitário Bettina Ferro de Souza (HUBFS), localizado no município de Belém, estado do Pará, no período de Abril de 2017 à Agosto de 2017, mediante a aprovação pelo Comitê de Ética em Pesquisa (número do protocolo; 2.026.577). O estudo é de caráter experimental, exploratório, com intenenção, estatístico analítico-descritivo e comparativo, de raciocínio quantitativo, seguindo uma abordagem transversal e prospectiva. 
No HUBFS, são realizadas em média 16 cirurgias semanais de exérese de pterígio, em pacientes provenientes de todo o Sistema Único de Saúde no Estado do Pará. Sendo, portanto, previsto um total aproximado de 256 pacientes na pesquisa, no entanto, devido a problemas logísticos o agendamento cirúrgico no período foi menor, além do período de férias de alguns cirurgiões, sendo realizadas em torno de 59 cirurgias no período, das quais apenas 38 cumpriram os critérios de inclusão.

Não foi realizada amostragem, visto ser estudo de população total. Foram incluídos na pesquisa: todos os pacientes portadores de pterígio submetidos a cirurgia de excisão no período da pesquisa. Foram excluídos da pesquisa: pacientes com idade inferior a 18 anos; pacientes portadores de distrofias corneanas; transplantados de córnea; bem como os que não aceitaram participar do estudo; e aqueles com quaisquer outras patologias do segmento posterior. Por ser um hospital referência em oftalmologia na região norte, o HUBFS possui um volume cirúrgico mensal na especialidade de em torno de 160 cirurgias oftalmológicas em geral, com uma equipe de 11 preceptores Oftalmologistas e 12 médicos residentes.

Todos os pacientes da presente pesquisa foram estudados segundo os preceitos da Declaração de Helsinque e do Código de Nuremberg, respeitadas as Normas de Pesquisa Envolvendo Seres Humanos (Res. CNS 466/12) do Conselho Nacional de Saúde somente após aprovação de anteprojeto pelo Núcleo de Pesquisa e Extensão de Medicina e Comissão de Ética da UEPA, sendo devidamente autorizado pelos pacientes e seus responsáveis. Foi obrigatória a assinatura dos representantes legais e dos pacientes nos Termo de Consentimento Livre e Esclarecido dos pacientes (TCLE). Quando o paciente não estava apto a assinar, utilizava-se somente a assinatura de seu representante legal. Não foram divulgados os nomes ou quaisquer meios de identificação dos participantes da pesquisa.

Os pacientes da pesquisa foram informados sobre seus objetivos e métodos, além de ter-lhes sido dado acesso livre aos resultados parciais e final da pesquisa, tendo ainda acesso ao endereço e telefone para contato dos pesquisadores, para que possam esclarecer dúvidas sobre o desenvolvimento do projeto. Além do mais, puderam se retirar da pesquisa a qualquer momento, dependendo de sua vontade, sem qualquer prejuízo para o seu tratamento no HUBFS. Não houve qualquer espécie de remuneração para os pacientes da pesquisa. No caso de haver qualquer dano ao paciente da pesquisa por conta do desenvolvimento do projeto, esses poderão requerer indenização cabível junto ao poder judiciário, segundo sua vontade.

A pesquisa foi baseada em protocolo próprio estabelecido pelos autores. A observação foi feita através de questionário sócio-demográfico com as variáveis sexo (Masculino ou Feminino), idade (anos), recidiva ou não; e de formulário para preenchimento médico com as variáveis: localização do pterígio (nasal, temporal, inferior e/ou superior), grau do pterígio (I - <2mm sobre a córnea, II - 2-4 mm sobre a córnea, III - >4 mm sobre a córnea, IV - cruzando a margem pupilar) (medida em mm (através da lâmpada de fenda) da distância limbo-ápice do pterígio), descrição de comorbidades, ceratometria automatizada (Valores ceratométricos dos dois maiores eixos), refratometria objetiva automatizada (grau esférico, cilíndrico e eixo), acuidade visual conforme optótipos de Snellen com e sem correção óptica, e observações como complicações cirúrgicas. A pesquisa foi divida em duas fases. Na fase 1 realizou-se a aplicação do questionário sócio-demográfico e formulário para preenchimento médico no pré-operatório, bem como preenchimento de TCLE. Seguido da cirurgia, no primeiro dia pós-operatório foram realizadas novas medidas dos itens: ceratometria automatizada, refratometraia objetiva automatizada e acuidade visual com e sem correção, bem como no sétimo e trigésimo pós-operatório. Na fase 2, os dados do questionário e formulário foram analisados conforme estatística descritiva, utilizando o Microsoft Excel $2017 \AA$ para cálculos e medidas de tendência central e dispersão para a idade, e de frequência absoluta e relativa para todos os demais dados. Comparou-se os parâmetros dos itens ceratometria automatizada, refratometraia objetiva automatizada e acuidade visual com e sem correção, entre os períodos, sendo calculadas as variações médias dos mesmos. Não foram aplicados testes de validação amostral por se tratar de um estudo com população total (WASSERSTEIN e LAZAR, 2016; FILHO et al.,2013).

Durante o desenvolvimento da pesquisa caso algum paciente apresentasse qualquer intercorrência médica decorrente ou não das atividades desenvolvidas no estudo, seria atendido nas dependências do HUBFS.

REAS/EJCH | Vol.Sup.22 | e847 | DOI: https://doi.org/10.25248/reas.e847.2019 Página 3 de 9 


\section{RESULTADOS}

A pesquisa incluiu um total de 38 pacientes, com idade média de 45,8 anos (Desvio-Padrão de $\pm 13,9$ anos), sendo $52,6 \%$ mulheres, em sua maioria com pterígio de localização nasal $(84,2 \%)$, com predomínio daqueles com Graus II e III, por fim somente 7,9\% eram pterígios recidivados (Tabela 1). Houve pequena melhora da acuidade visual corrigida dos pacientes submetidos a cirurgia de pterígio, independente do grau de pterígio da pesquisa (Tabela 3), que encontrou uma acuidade visual corrigida média inicial de 0,59 $\pm 0,28$ e pós-operatória final de $0,85 \pm 0,2216$, sendo a melhora mais proeminente no grupo de Graus III e IV de pterígio na amostra presente. O tipo de astigmatismo inicial mais prevalente no estudo foi o astigmatismo com-a-regra (39.5\%) e a ametropia inicial mais frequente encontrados pacientes foi o astigmatismo misto e 0 astigmatismo hipermetrópico composto, ambos com $23,7 \%$ cada (Tabela 2). As diferenças refratométricas objetivas médias antes e após a exérese de pterígio foram de 0,15 a 4,45 dioptrias para o astigmatismo corneano, 1,25 a 4,25 dioptrias para o grau cilíndrico, 0,75 a 4,75 dioptrias para o grau esférico, 1,18 a 6,88 dioptrias para o equivalente esférico (Tabela 4), e 17,8 a 105 graus para o eixo cilíndrico (Tabela 5), variando ao longo do tempo em curva descendente e conforme o grau de pterígio, sem estabilizar-se dos $7^{\circ}$ ao 30 으 dia pós-operatório.

Tabela 1 - Variáveis Sócio-Epidemiológicas em pacientes submetidos a exérese de Pterígio no Hospital Bettina Ferro de Souza no período de Abril à Agosto de 2017.

\begin{tabular}{cccc}
\hline & Variável & N & $\%$ \\
\hline Gênero & & & \\
& Masculino & 18 & 47,4 \\
& Feminino & 20 & 52,6 \\
\hline & TOTAL & 38 & - \\
\hline
\end{tabular}

Recidiva do Pterígio

\begin{tabular}{lccc} 
& Sim & 3 & 7,9 \\
& Não & 35 & 92,1 \\
\hline Localização do Pterígio & TOTAL & 38 & - \\
\hline & Nasal & 32 & 84,2 \\
& Temporal & 1 & 2,6 \\
& Nasal/Temporal & 5 & 13,2 \\
\hline & TOTAL & 38 & - \\
\hline
\end{tabular}

Grau do Pterígio

\begin{tabular}{cccc} 
& I & 2 & 5,3 \\
& II & 17 & 44,7 \\
\hline III & 18 & 47,4 \\
\hline & IV & 1 & 2,6 \\
\hline
\end{tabular}

Fonte: Formulário da Pesquisa (Apêndice 3). 
Tabela 2 - Variáveis refratométricas em pacientes submetidos a exérese de Pterígio no Hospital Bettina Ferro de Souza no período de abril a agosto de 2017.

\begin{tabular}{lll}
\hline Variável & $\mathbf{N} \%$ \\
\hline
\end{tabular}

Tipo de Astigmatismo Inicial

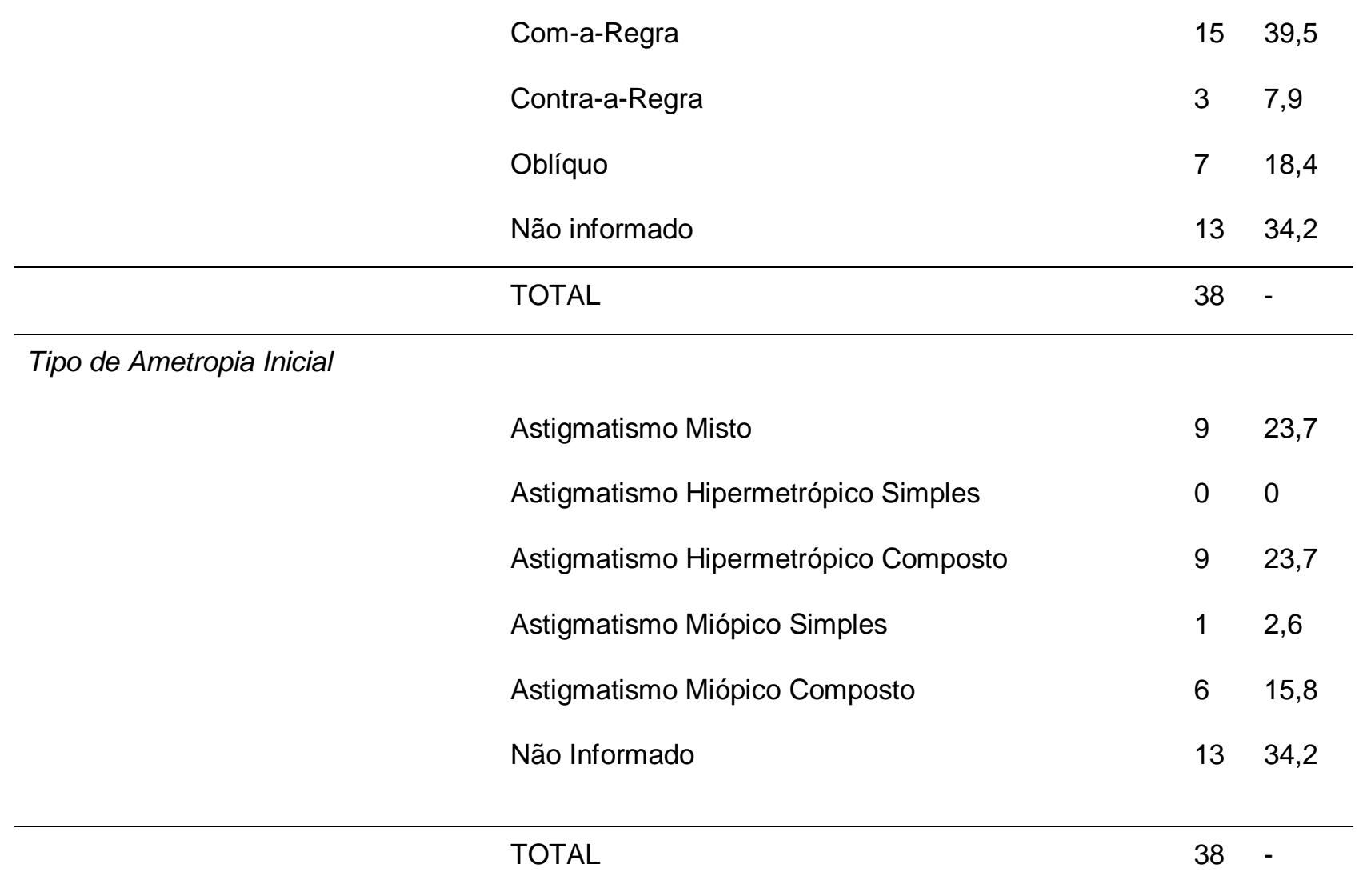

Fonte: Formulário da Pesquisa (Apêndice 3).

Tabela 3 - Acuidade Visual com Correção em pacientes submetidos a exérese de Pterígio no Hospital Bettina Ferro de Souza no período de Abril à Agosto de 2017.

\begin{tabular}{lccc}
\hline & Variável & Média & Desvio-Padrão \\
\hline Pterígio Graus I e II & Inicial & 0,89 & $\pm 0,25$ \\
Pterígio Graus III e IV & 30 dias após Exérese de Pterígio & 0,93 & $\pm 0,15$ \\
& Inicial & 0,65 & $\pm 0,33$ \\
& 30 dias após Exérese de Pterígio & 0,73 & $\pm 0,42$ \\
\hline
\end{tabular}

Legenda: AVsc: Acuidade Visual sem Correção. AVcc: Acuidade Visual com Correção.

Fonte: Formulário da Pesquisa (Apêndice 3). 
Tabela 4 - Variação Média da Refratometria em Dioptrias conforme o Grau do Pterígio e conforme o intervalo de tempo pós-operatório em pacientes submetidos a exérese de Pterígio no Hospital Bettina Ferro de Souza no período de Abril à Agosto de 2017.

\begin{tabular}{|c|c|c|c|c|}
\hline $\begin{array}{l}\text { Variáveis } \\
\text { Refratométricas }\end{array}$ & $\begin{array}{l}\text { Grau do Pterígio } \\
\text { Excisado }\end{array}$ & Pré-Op. ao 1 PO & $\begin{array}{l}\text { 19PO ao } \\
7^{\circ} P O\end{array}$ & $\begin{array}{l}7^{\circ} P O \text { ao } \\
30^{\circ} P O\end{array}$ \\
\hline \multirow{3}{*}{$\begin{array}{l}\text { Astigmatismo } \\
\text { Corneano }\end{array}$} & \multicolumn{4}{|c|}{$\begin{array}{l}\text { *Variação Média em Dioptrias (Desvio- } \\
\text { Padrão) }\end{array}$} \\
\hline & Graus I e II & $0,15( \pm 0,05)$ & $1,68( \pm 1,21)$ & $\begin{array}{c}1,08 \\
( \pm 1,02)\end{array}$ \\
\hline & Graus III e IV & $4,45( \pm 0,99)$ & NA & $\begin{array}{c}0,68 \\
( \pm 0,29)\end{array}$ \\
\hline \multirow[t]{2}{*}{ Grau Cilíndrico } & Graus I e II & $1,25( \pm 0,64)$ & $1,30( \pm 0,91)$ & $\begin{array}{c}1,17 \\
( \pm 1,38)\end{array}$ \\
\hline & Graus III e IV & $4,25( \pm 0,95)$ & NA & $\begin{array}{c}0,55 \\
( \pm 0,38)\end{array}$ \\
\hline \multirow[t]{2}{*}{ Grau Esférico } & Graus I e II & $0,75( \pm 0,46)$ & $0,90( \pm 0,58)$ & $\begin{array}{c}0,56 \\
( \pm 0,47)\end{array}$ \\
\hline & Graus III e IV & $4,75( \pm 1,06)$ & $0,25( \pm 0,06)$ & $\begin{array}{c}1,10 \\
( \pm 0,79)\end{array}$ \\
\hline \multirow[t]{2}{*}{ Equivalente Esférico } & Graus I e II & $1,18( \pm 0,76)$ & $1,40( \pm 1,03)$ & $\begin{array}{c}0,80 \\
( \pm 0,84)\end{array}$ \\
\hline & Graus III e IV & $6,88( \pm 1,54)$ & $0,25( \pm 0,06)$ & $\begin{array}{c}1,33 \\
( \pm 0,93)\end{array}$ \\
\hline
\end{tabular}

Legenda: Pré-op. = Pré-operatório; PO = Pós-operatório; NA = Não Avaliado.

Fonte: Formulário da Pesquisa (Apêndice 3).

Tabela 5 - Variação Média da Refratometria em Dioptrias conforme o Grau do Pterígio e conforme o intervalo de tempo pós-operatório em pacientes submetidos a exérese de Pterígio no Hospital Bettina Ferro de Souza no período de Abril à Agosto de 2017.

\begin{tabular}{|c|c|c|c|c|}
\hline \multirow[b]{3}{*}{ Variável Refratométrica } & \multicolumn{4}{|l|}{ Grau do } \\
\hline & Pterígio & Pré-Op. ao $1^{\circ}$ & $1{ }^{\circ} P O$ ao & $7^{\circ} P O$ ao \\
\hline & Excisado & PO & $7^{\circ} P O$ & $30^{\circ} P O$ \\
\hline \multicolumn{5}{|c|}{ *Variação Média em Graus (Desvio-Padrão) } \\
\hline \multirow[t]{2}{*}{ Eixo Cilíndrico } & Graus I e II & $17,80( \pm 14,44)$ & $26,40( \pm 19,33)$ & $14,40( \pm 21,06)$ \\
\hline & Graus III e IV & $105,00( \pm 23,45)$ & $52,00( \pm 11,61)$ & $40,00( \pm 23,01)$ \\
\hline
\end{tabular}

Legenda: Pré-op. = Pré-operatório; PO = Pós-operatório; NA = Não Avaliado.

Fonte: Formulário da Pesquisa (Apêndice 3). 


\section{DISCUSSÃO}

Sendo o pterígio morbidade de alta prevalência na população amazônica (9,3\%) (BRANCO BC, 2000), se vislumbra a necessidade de discussão deste tema. Quanto aos dados relativos a faixa etária dos pacientes da pesquisa, foram condizentes com a literatura, conforme VIEGAS MTC et al. (2016), que descreveu uma faixa etária principalmente distribuída dos 20 aos 49 anos.

A técnica cirúrgica utilizada para excisão do pterígio na pesquisa foi a rotação de retalho conjuntival, executada por 4 diferentes cirurgiões. Em relação as variadas de técnicas para este feito, não existe diferença entre elas quanto ao efeito provocado sobre as ametropias encontradas (KHEIRKHAH A et al., 2012) (LEITE DMS, 2010), pois variam entre si quanto ao componente conjuntival da técnica, principalmente. Não houve diferença expressiva entre os gêneros dos pacientes da pesquisa, condizendo com as publicações anteriores (LEITE DMS, 2010).

O astigmatismo associado ao pterígio é a favor da regra, devido as forças mecânicas que geram aplanamento do meridiano horizontal, bem como alterações da superfície lacrimal provocando distúrbios refrativos (FERRAZ FHS et al., 2002; KHEIRKHAN A et al., 2012), sendo encontrada na pesquisa atual prevalência de $39,4 \%$ deste tipo de astigmatismo entre os pacientes previamente a exérese do pterígio, os demais tipos de astigmatismo encontrado podem ser atribuídas a características refratométricas do paciente independentes deste agravo de saúde. A população estudada apresentou uma taxa de casos recidivados a serem reabordados dentro do previsto na literatura (7,9\%), seguindo o demonstrado por LEITE DMS (2017) que sugere taxas de $0 \%$ a $39 \%$. A maior parte dos pterígios apresentou localização nasal $(84,2 \%)$, visto que é a região de maior acometimento (ALVES MR, 1999). Os pterígios dos graus III e II predominaram $(47,4 \% \mathrm{e}$ 44,7\%, respectivamente) (FERRAZ FHS et al., 2012).

Houve pequena melhora da acuidade visual corrigida dos pacientes da pesquisa submetidos a cirurgia de pterígio, independente do grau de pterígio, o que vai de acordo com YAGMUR et al (2005) que encontrou uma acuidade visual corrigida média inicial de $0,59 \pm 0,28$ e pós-operatória final de $0,85 \pm 0,2216$, sendo a melhora mais proeminente no grupo de Graus III e IV de pterígio (YAGMUR MMD et al., 2005). Cita-se que quanto maior o tamanho da lesão maior, de fato, a ametropia induzida, sendo que ela pode ser parcialmente corrigida com a cirurgia (AVISAR R et al., 2000).

Quanto a variação do astigmatismo após a excisão do pterígio, FERRAZ et al (2002) encontrou-se variação máxima de 1,50 dioptrias após 1 mês de seguimento, variações superiores foram vistas nesta pesquisa, onde em média, os pterígios de graus I e II excisados tiveram redução de 1,25 dioptrias $\pm 0,64$, e aqueles com graus III e IV chegaram a ter variação de 4,25 dioptrias $\pm 0,95$, valores somente para as variações 1 dia após a excisão, sendo ainda maiores quando considerada a variação ao longo de 1 mês. O mesmo pode ser dito pelos dados encontrados por MAHESHWARI S (2007). No caso do estudo de POPAT et al (2014), a variação no astigmatismo foi similar a presente pesquisa, talvez devido a prevalência elevada de pterígios de graus maiores em seu estudo, este último encontrou variações de até 4.03 dioptrias no $1^{\circ}$ pós-operatório em pterígios de Graus I e II, e de 3,87 dioptrias em pterígios de graus III e IV, valores que na presente foram, ao contrário disto, maiores nos pterígios de graus maiores, já quando comparados os grupos do primeiro ao sétimo dia pós-operatório e do sétimo ao trigésimo pós-operatório, o autor supracitado encontrou dados similares a pesquisa atual, com pterígios maiores causando maior variação no astigmatismo, as amostras dos dois estudos são similares em relação ao perfil epidemiológica e técnica cirúrgica utilizadas, diferindo somente em relação a localização geográfica de seus participantes.

Em relação a ceratometria de córnea, que correlaciona-se com o componente corneano do astigmatismo,

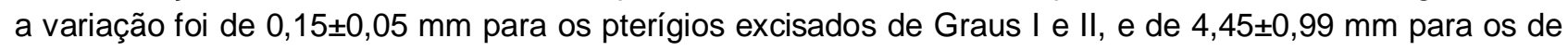
Graus III e IV, dados que se assimilam aos de ERRAIS et al. (2008) e YAGMUR et al. (2005), e MAHESHWARI $S$ (2007)' que encontraram córneas mais curvas após a cirurgia. FERRAZ et al. (2002) associa o componente corneano do astigmatismo maior nos casos de graus III e IV, mas também uma maior variação deste após a cirurgia de excisão, já TOMIDOKORO et al. (1999) refere que estas alterações são somente parcialmente corrigidas pela cirurgia. 
Quanto ao eixo do astigmatismo, houveram variações no seu valor em todos os intervalos pós-operatórios investigados, sendo mais importante a variação nos pterígios de Graus III e IV, divergindo do proposto por ERRAIS et al. (2008), que não encontrou variações neste componente refratométrico.

STERN e ALISON (1999) associam pterígios com diâmetro horizontal excedendo $45 \%$ do diâmetro corneano a efeitos significativos a refração, propondo indicação cirúrgica a partir deste valor.

Algumas vezes, o astigmatismo final pode ser aumentado, ao invés de reduzido com a cirurgia, isto ocorreu em 2 pacientes na presente pesquisa, e foi descrito também por BAHAR et al. (2004) e TOMIDOKORO et al. (1999) ,sendo provavelmente associado ao fato de haverem características próprias da córnea do paciente, subjacentes e independentes da cirurgia.

Nesta pesquisa, os pacientes não foram avaliados após o $1^{\circ}$ mês pós-operatório, pois a literatura revela que não há variação expressiva após esse período (MAHESHWARI S, 2007). Concordando com KEIRKHAN et al. (2012), que não encontrou alterações significativas no astigmatismo anterior e posterior da córnea após 1 mês de excisão, nem mesmo após 6 meses. Já POPAT et al. (2014), assim como TOMIDOKORO (2000), encontraram estabilização refratométrica em 45 dias de pós-operatório.

Nenhuma das pesquisas supracitadas comparou variações do componente esférico ou mesmo do equivalente esférico antes e após a excisão do pterígio, sendo que nesta pesquisa foram encontradas modificações expressivas nestes parâmetros também, sendo eles essenciais para a prescrição da correção óptica, não podem ser desconsiderados (Tabela 4).

\section{CONCLUSÃO}

Na presente pesquisa, constatamos a importância da exérese do pterígio na melhora da acuidade visual do paciente, com redução expressiva da ametropia, principalmente nos casos mais avançados (graus III e IV). Portanto, quando pensamos em indicação cirúrgica por motivo refrativo no pterígio, visando obter uma melhora da acuidade visual do paciente, devem ser considerados os pterígios de graus III e IV. Como contribuição para comunidade, o estudo evidenciou que não há benefício na prescrição de óculos antes de 30 dias de pós operatório, haja vista que não há estabilização refrativa antes deste período, ficando constatado que o melhor período para prescrição de óculos é acima de 30 a 45 dias da cirurgia. A pesquisa auxiliou a corroborar dados já evidenciados na literatura revisada, objetificando a melhora da acuidade visual, bem como evitando prescrições refratoméricas inadequadas que poderiam causa desconforto e/ou insatisfações futuras nos pacientes.

\section{REFERÊNCIAS}

1. ALVES MR. Pterígio In: Conjuntiva Cirúrgica. São Paulo: Editora Rocca, 1999. 59-66p.

2. AVISAR R, et al. Pterygium-induced corneal astigmatism. Israel Med Assoc J 2000;2:14-5.

3. BAHAR, IM, et al. Effect of Pterygium Surgery on Corneal Topography: A Prospective Study. The Journal of Cornea and External Disease. March 2004 - Volume 23 - Issue 2 - pp 113-117.

4. BRANCO, BC. Pesquisa Operacional Em Saúde Ocular Em Região De Alto Analfabetismo Da Amazônia: Detecção Dos Agravos E Intervenção Nas Ametropias. Universidade Federal de São Paulo (UNIFESP), São Paulo: [s.n.], 2000. 111 p. tabgraf 2000.

5. CHOURASIA P, et al. Comparison of astigmatism before and after pterygium surgery. Int $\mathrm{J}$ Health Sci Res. 2014;4(3):97-102.

6. COUTTS SJ, COOMBES A. Pterygium: prevalence and severity in an Amazonian ophthalmic setting, Brazil. Rev. bras.oftalmol., Rio de Janeiro, v. 71, n. 6, p. 372-376, Dec. 2012. 
7. ERRAIS K, et al. Effect of pterygium surgery on corneal topography. European Journal of Ophthalmology. Department of Ophthalmology, Charles Nicolle University Hospital, Tunis - Tunisia, Vol. 18 no. 2, 2008 / pp. 177 181.

8. FERRAZ FHS, et al. Pterígio e alterações da curvatura corneana. Arq. Bras. Oftalmol., São Paulo, v. 65, n. 5, p. 533-536, Sept. 2002.

9. GUMUS K, et al. Effect of Pterygia on Refractive Indices, Corneal Topography, and Ocular Aberrations. Cornea Volume 30, Number 1, January 2011.

10. KHEIRKHAH A, et al. Effects of pterygium surgery on front and back corneal astigmatism. Canadian Journal of Ophthalmology / Journal Canadian d'Ophtalmologie, Volume 47, Issue 5, October 2012, Pages 423-428.

11. KUJUR R, et al. To study the effect of pterygium excision on corneal curvature and contrast sensitivity. Int J Med Res Rev 2015;3(11):1281-1287. doi: 10.17511/ijmrr.2015.i11.233.

12. LEITE DMS. Estudo comparativo da técnica de transplante autólogo de conjuntiva quanto ao local de retirada do retalho conjuntival para correção de pterígio primário. Dissertação (Mestrado em Ciências da Saúde) Universidade de Brasília, 67 f . Brasília, 2010.

13. MAHESHWARI S. Pterygium-induced corneal refractive changes. Indian Journal of Ophthalmology. Sep-Oct; 2007; v. 55(5): p.383-386.

14. MISRA S, et al. A prospective study of pterygium excision \& conjunctival autograft with human fibrin tissue adhesive: effects on vision, refraction \& corneal topography. Asia-Pacific Journal of Ophthalmology, 2014 July/August; $3(4): 202-206$.

15. POPAT KB, et al. A study on changes in keratometry readings and astigmatism induced by pterygium before and after pterygium excision surgery. Journal of Research in Medical and Dental Science. Vol 2, No 3 . | July September 2014.

16. STERN GAMD, LIN AMD. Effect of Pterygium Excision on Induced Corneal Topographic Abnormalities. The Journal of Cornea and External Disease. January 1998 - Volume 17 - Issue 1.

17. TOMIDOKORO A, et al. Quantitative analysis of regular and irregular astigmatism induced by pterygium. Cornea, 1999;18:412-5.

18. VIEGAS MTC et al. Avaliação da qualidade de vida em portadores de pterígio ocular no município de Palmas. Revista de Atenção à Saúde (antiga Rev. Bras. Ciên. Saúde), v. 14, n. 47, p. 5-11, 2016.

19. WASSERSTEIN RL, LAZAR NA. Statement on p-Values: Context, Process, and Purpose, The American Statistician, 70:2, 129-133, 2016.

20. YAGMUR MMD, et al. Visual acuity and corneal topographic changes related with pterygium surgery. Journal of Refractive Surgery. March 2005 - Volume 21 Issue 2: 166-170. 